\title{
The application of extended modified Lambert Beer model for measurement of blood carboxyhemoglobin and oxyhemoglobin saturation
}

\author{
Audrey Huong* \\ Department of Electronic Engineering \\ Faculty of Electrical and Electronic Engineering \\ University Tun Hussein Onn Malaysia \\ Batu Pahat 86400, Johor, Malaysia \\ audrey@uthm.edu.my \\ Xavier Ngu \\ Department of Communication Engineering \\ Faculty of Electrical and Electronic Engineering \\ University Tun Hussein Onn Malaysia \\ Batu Pahat 86400, Johor, Malaysia
}

Received 16 August 2013

Accepted 6 December 2013

Published 5 February 2014

\begin{abstract}
This work presents the use of extended Modified Lambert Beer (MLB) model for accurate and continuous monitoring of percent blood carboxyhemoglobin (COHb) (SCO) and oxyhemoglobin $(\mathrm{OxyHb})$ saturation $\left(\mathrm{SO}_{2}\right)$ via a fitting procedure. This quantification technique is based on the absorption characteristics of hemoglobin derivatives in the wavelength range of 520-600 nm to give the best estimates of the required parameters. A comparison of the performance of the developed model and MLB law is made using attenuation data from Monte Carlo simulations for a two-layered skin model. The results revealed a lower mean absolute error of $0.4 \%$ in the values estimated by the developed model as compared to $10 \%$ that is given by the MLB law. This study showed that the discussed approach is able to provide consistent and accurate measurement of blood $\mathrm{SO}_{2}$ and $\mathrm{SCO}$ across different skin pigmentations suggesting that it may potentially be used as an alternative means for clinical diagnosis of carbon monoxide $(\mathrm{CO})$ poisoning.
\end{abstract}

Keywords: Blood carboxyhemoglobin saturation; carbon monoxide poisoning; blood oxyhemoglobin saturation; modified Lambert Beer law.

${ }^{*}$ Corresponding author.

This is an Open Access article published by World Scientific Publishing Company. It is distributed under the terms of the Creative Commons Attribution 3.0 (CC-BY) License. Further distribution of this work is permitted, provided the original work is properly cited. 


\section{Introduction}

The concentration of carbon monoxide $(\mathrm{CO})$ in blood or carboxyhemoglobin $(\mathrm{COHb})$ is the key parameter used to evaluate one's CO toxicity level after exposure to the flammable, toxic gas. The elevated level percent of $\mathrm{COHb}$ in blood to $60 \%$ can prove fatal ${ }^{1}$ as $\mathrm{CO}$ molecules, which have a higher tendency to bind with hemoglobin, reduce the concentration of oxygen bound to hemoglobin (known as oxyhemoglobin (OxyHb)). The accurate measurement of both of these parameters is, therefore, essential so that suitable oxygen therapy can be administered in response to the different severity of $\mathrm{CO}$ poisoning. Nonetheless the conventional technique used for accurate measurement of percent blood $\mathrm{COHb}$ saturation ( $\mathrm{SCO}$ ) is limited to those that involve collecting and analyzing blood sample, which is impractical for continuous monitoring of one's blood CO level. Non-invasive and continuous measurement of $\mathrm{COHb}$ can be provided by pulse CO-oximeter but this device is shown to produce large error in the estimated value of $14 \% .^{2}$ Meanwhile, fast and non-invasive measurement of arterial blood OxyHb saturation $\left(\mathrm{SO}_{2}\right)$ can be provided by pulse oximetry, but its high accuracy is limited to $\mathrm{SO}_{2}$ greater than $70 \%$. Techniques that are able to provide fast and accurate measurement of percent saturation of oxygen and $\mathrm{CO}$ in blood are of significant value, especially to individuals who are occupationally exposed to incomplete combustion of organic materials such as that of coal-fired power plant operators and firefighters.

Although the absolute concentration measurement of an absorber using signals from continuous wave (CW) spectroscopy is only feasible if the pathlength of photons travelled in the medium is known, ${ }^{3}$ for example, using the measurement of temporal point spread function of light traversing a scattering medium, the percentage of hemoglobin components can be deduced using light attenuation measured from CW spectroscopy performed on skin. ${ }^{4}$ The use of CW spectroscopic technique allows the non-invasive measurement of fractional concentration of an absorbing component by means of fitting using either analytic models or a library of data simulated using Monte Carlo method or diffusion model. ${ }^{4,5}$ Amongst the commonly used analytic models is Modified Lambert Beer (MLB) law that assumes a linear relationship between light absorption and attenuation. In addition to the fitting method, the required parameter is also commonly solved via simultaneous solution of this linear model. The relationship between light attenuation and absorption in blood is, however, a nonlinear and multi-valued function owing to the wavelength dependent scattering properties of different skin layers. This results in poor accuracy of the value estimated by the MLB. Other more complex models such as power law model developed from radiative transfer equation and cubic attenuation model are designed to provide a more accurate estimate of this value. ${ }^{4,6}$ However, their application is limited. Besides, because of the high melanin absorption and high scattering of skin tissue at the visible wavelengths, many of these workers preferred working with signals in the near infrared range.

This paper presents the use of an extended MLB model to extract the percentage saturation of $\mathrm{COHb}$ and $\mathrm{OxyHb}$ via a fitting algorithm. The performance of this model is evaluated using data given by Monte Carlo simulations as actual fractional concentrations of absorbers are known. The focus of this study is on signals measured across visible range as this gives greater flexibility in the selection of equipment during experiment, and this work aims to estimate both blood $\mathrm{SCO}$ and $\mathrm{SO}_{2}$ non-invasively using data collected from optical spectroscopy performed on skin surface without attempting to solve fractional concentration of deoxyhemoglobin ( $\mathrm{Hb})$.

\section{Methods}

\subsection{Mathematical model of skin and light propagation simulations}

Skin is a complex structure with inhomogeneous distribution of tissues, capillaries and blood vessels. Many previous studies have divided skin into three distinctive layers, namely, epidermis, dermis and subcutaneous layer. Recent efforts to obtain a closer approximation to the actual skin include modeling of skin as a nine layer medium having different optical properties. In this work, a two-layered skin model similar to that used in Yudovsky and Pilon ${ }^{7}$ is established, wherein the upper layer is an infinite slab with a commonly reported thickness of $100 \mu \mathrm{m}^{8}$ supported by a semi-infinite bottom layer. These upper and bottom layers are taken to be bloodless epidermis and blood-rich dermis layers, respectively, and are modeled to have the optical properties of the corresponding medium. Subcutaneous layer has not been considered in this skin model owing to the limit 
of light penetration depth of approximately $2 \mathrm{~mm}$ in skin. ${ }^{4}$ Even though the medium's refractive index is a wavelength dependent function, this variation is small in the considered visible wavelength range ${ }^{9}$ and so is assumed as $n=1.4$ for both layers.

Since the thickness of the epidermis is considerably lesser than that of the dermis, both of these layers have the same wavelength dependent reduced scattering coefficients. This is a result of the negligible differences observed on the simulated data when other scattering coefficients are used for epidermis layer. ${ }^{10}$ The wavelength dependent $\mu_{\mathrm{s}}^{\prime}$ values used in this work in Fig. 1 are taken from the results of Staveren et al. ${ }^{11}$ on intralipid $10 \%$ as the reported $\mu_{\mathrm{s}}^{\prime}$ range and monotonic variation in $\mu_{\mathrm{s}}^{\prime}$ with wavelength are similar to that of biological tissues presented in other works. ${ }^{12}$ Light absorption by melanin in the assumed blood-free epidermis, $\mu_{\mathrm{a}_{\mathrm{m}}}$, is taken from Meglinski and Matcher, ${ }^{8}$ wherein changes in $\mu_{\mathrm{a}_{\mathrm{m}}}$ with wavelength, $\lambda$ (in nanometers), can be approximated by ${ }^{8}$ :

$$
\mu_{\mathrm{a}_{\mathrm{m}}}=5 \times 10^{9} \lambda^{-3.33}\left(\mathrm{~mm}^{-1}\right) .
$$

The epidermal absorption coefficient, $\mu_{\mathrm{a}_{\text {epi. }} \text {, is }}$ given by the product of $\mu_{\mathrm{a}_{\mathrm{m}}}$ times volume fraction of melanosomes in epidermis. The volume fraction of melanosomes, $f_{\mathrm{v} \cdot \mathrm{mel}}$, ranges from $1-3 \%$ for a fair skinned person and up to $40 \%$ for darkly pigmented skin. ${ }^{13}$ The $\mu_{\mathrm{a}_{\mathrm{epi}}}$ values given from $\mu_{\mathrm{a}_{\mathrm{m}}}$ in Eq. (1) and $f_{\mathrm{v} \text {-mel }}$ of $40 \%$ chosen here for demonstration purposes are shown in Fig. 1.

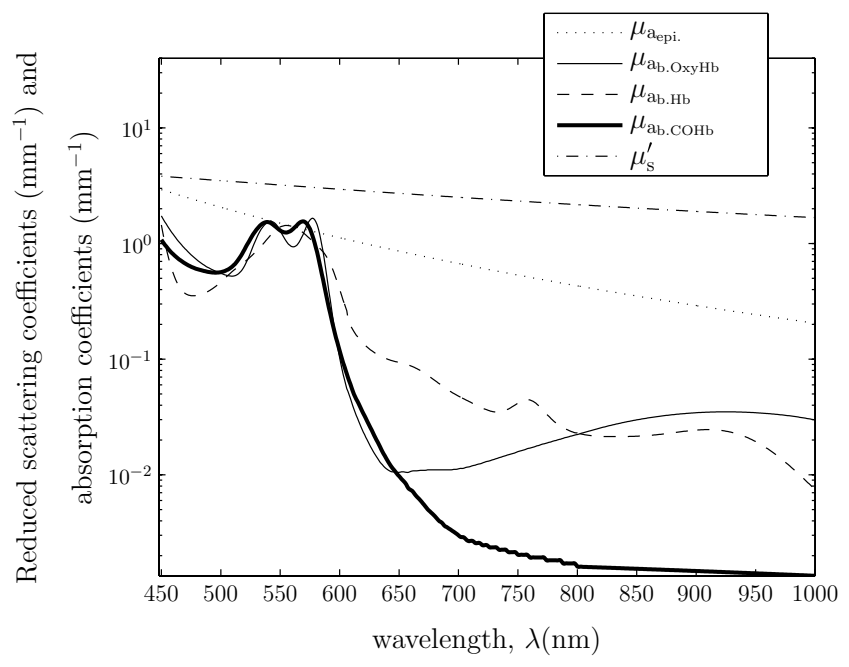

Fig. 1. Reduced scattering coefficients, $\mu_{\mathrm{s}}^{\prime}$, and epidermal absorption coefficients $\left(\mu_{\mathrm{a}_{\mathrm{epj}}}\right)$, absorption coefficients of $\mathrm{OxyHb}$

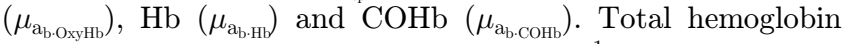
concentration is taken to be $268 \mu \cdot \mathrm{mol} \cdot \mathrm{L}^{-1}$.
Capillaries and blood vessels carrying hemoglobin molecules absorb light in the dermis layer, with blood $\mathrm{OxyHb}, \mathrm{Hb}$ and $\mathrm{COHb}$ taken here as three absorbers in this medium. The total light absorption in the dermis, $\mu_{\mathrm{a}_{\mathrm{b}}}(\lambda)$, is given by the summation of the product of concentration, $C$, and wavelength dependent extinction coefficient, $\varepsilon(\lambda)$, of each absorbing species (indicated by subscript notation) as follows:

$$
\begin{aligned}
\mu_{\mathrm{ab}_{\mathrm{b}}}(\lambda)= & \varepsilon_{\mathrm{OxyHb}}(\lambda) C_{\mathrm{OxyHb}}+\varepsilon_{\mathrm{Hb}}(\lambda) C_{\mathrm{Hb}} \\
& +\varepsilon_{\mathrm{COHb}}(\lambda) C_{\mathrm{COHb}} .
\end{aligned}
$$

Given that $T=C_{\mathrm{OxyHb}}+C_{\mathrm{Hb}}+C_{\mathrm{COHb}}$ is the total hemoglobin concentration, Eq. (2) can be written as Eq. (3). Next, rearranging Eq. (3) gives Eq. (4), wherein $\mathrm{SO}_{2}$ and $\mathrm{SCO}$ in Eq. (4) are blood $\mathrm{OxyHb}$ and $\mathrm{COHb}$ saturation, respectively, given by:

$$
\begin{aligned}
& \mathrm{SO}_{2}=\frac{C_{\mathrm{OxyHb}}}{T}, \\
& \mathrm{SCO}=\frac{C_{\mathrm{COHb}}}{T},
\end{aligned}
$$

$$
\begin{aligned}
\mu_{\mathrm{ab}_{\mathrm{b}}}(\lambda)= & \varepsilon_{\mathrm{OxyHb}}(\lambda) C_{\mathrm{OxyHb}}+\varepsilon_{\mathrm{COHb}}(\lambda) C_{\mathrm{COHb}} \\
& +\varepsilon_{\mathrm{Hb}}(\lambda)\left(T-C_{\mathrm{OxyHb}}-C_{\mathrm{COHb}}\right), \\
\mu_{\mathrm{ab}_{\mathrm{b}}}(\lambda)= & \left(\left(\varepsilon_{\mathrm{OxyHb}}(\lambda)-\varepsilon_{\mathrm{Hb}}(\lambda)\right) \mathrm{SO}_{2}+\left(\varepsilon_{\mathrm{COHb}}(\lambda)\right.\right. \\
& \left.\left.-\varepsilon_{\mathrm{Hb}}(\lambda)\right) \mathrm{SCO}+\varepsilon_{\mathrm{Hb}}(\lambda)\right) T .
\end{aligned}
$$

Using the total hemoglobin concentration of $T=268 \mu \cdot \mathrm{mol} \cdot \mathrm{L}^{-1}$, which is calculated from the reported dermis blood volume fraction of $5 \%,{ }^{13}$ and the extinction coefficient of different hemoglobin derivatives reported by Zijlistra, ${ }^{14}$ the absorption of each absorber in dermis layer is shown in Fig. 1.

The distinctive differences in the absorption of hemoglobin components can be observed in the wavelength range of $520-600 \mathrm{~nm}$ in Fig. 1. The quantification approach employed in this study depends on this absorption spectra and a fitting procedure that will be described in Sec. 2.2 to give the best guess of the required parameters, so this paper works with signals measured across this wavelength range to find the value of $\mathrm{SO}_{2}$ and $\mathrm{SCO}$.

This work used attenuation data generated using Monte Carlo simulation code described in Chang et $a l .{ }^{15}$ to verify the accuracy of value estimated by the developed analytic model and to compare the performance of the corresponding model with that 
of the MLB law. For each $\mu_{\mathrm{s}}^{\prime}$ value in the wavelength range of $520-600 \mathrm{~nm}$ in Fig. 1, the behavior of light propagate in the medium is simulated after $1 \times 10^{6}$ photons are launched normally into the medium. Photons propagate with optical pathlength greater than $100 \mathrm{~mm}$ are discarded owing to their low, if any, contribution to the overall measured intensity. Since the skin is modeled in this work as an infinitely thick medium, the simulation data is derived from reflectance mode measurement. The detection of photons is via an annular detector with inner and outer detection radius of $2 \mathrm{~mm}$ and $4 \mathrm{~mm}$, respectively, from the light source. This detector is placed directly at the skin surface.

For demonstration purposes, 16 arbitrarily selected combinations of percent $\mathrm{SO}_{2}$ and $\mathrm{SCO}, \mathrm{c}_{x}$, used to give dermis total absorption coefficient shown in Eq. (4) are tabulated in Table 1, where the subscript $x$ represents the combination index. In addition, an investigation of the consistency in the performance of the developed model using data measured from populations with different skin pigmentation is by considering $f_{\mathrm{v} \cdot \mathrm{mel}}$, of $2 \%$ and $40 \%$ in this study. The effects of these dermal and epidermal absorption are incorporated post simulation into Monte Carlo simulated data according to Beer's law to speed up the simulation process.

\subsection{Extended modified Lambert Beer model and fitting procedure}

The common practice employed in solving an absorber's fractional concentration value is by the simultaneous solution of MLB in Eq. (5) or via the fitting of data simulated from light propagation model to the measured spectra. ${ }^{5,16}$ This paper proposes the use of an attenuation model extended from MLB model (hence the name extended MLB) shown in Eq. (6) to find the value of $\mathrm{SO}_{2}$ and $\mathrm{SCO}$ in Eq. (4) by means of fitting to the measured spectra

$$
\begin{gathered}
A(\lambda)=G_{0}+\mu_{\mathrm{a}} d_{0}, \\
A(\lambda)=G_{0}+\mu_{\mathrm{a}} d_{0}+G_{1} \lambda+\lambda \exp \left(-\mu_{\mathrm{a}} d_{1}\right) .
\end{gathered}
$$

Here $\mu_{\mathrm{a}}$ represents light absorption. The first and second terms in Eq. (6) are from the MLB describing the light attenuation offset and absorption dependent light attenuation, respectively, with the parameter $d_{0}$ normally taken to be "light pathlength".

Since both scattering coefficients and light absorption by epidermis decrease approximately linearly with wavelength in the considered wavelength range in Fig. 1, this work assumed light attenuation due to scattering process and light absorption in the epidermis is represented by parameter $G_{1} \lambda$ in Eq. (6) so that $\mu_{\mathrm{a}}$ in second and last term of this equation is given from the absorption by blood rich dermis expressed in Eq. (4). This gives an expression of light attenuation as a function of blood $\mathrm{OxyHb}$ and $\mathrm{COHb}$ saturation in Eq. (7) with the symbol $\Delta \varepsilon_{1}$ and $\Delta \varepsilon_{2}$ in Eq. (7) is given by $\varepsilon_{\mathrm{OxyHb}}(\lambda)-\varepsilon_{\mathrm{Hb}}(\lambda)$ and $\varepsilon_{\mathrm{COHb}}(\lambda)-\varepsilon_{\mathrm{Hb}}(\lambda)$, respectively. Meanwhile the exponential term is included in this model to represent the light attenuation as a complex function of dermal light scattering, which is assumed to yield changes in "light path-length", $d_{1}$, and absorption in the dermis.

The value of $\mathrm{SCO}$ and $\mathrm{SO}_{2}$ is found by fitting this model to the simulated data using fminsearch function in MATLAB. This optimization method does not apply constraints to the value of these fitting parameters but it will seek for a new value of these parameters according to the size of error between the value given from the employed analytic model and the real attenuation, $\Delta E$, during each iteration. This fitting process is terminated when either the mean absolute $\Delta E$ is less than $1 \times 10^{-20}$ or the iteration process has exceeded 1000, when the optimal value of $\mathrm{SCO}$ and $\mathrm{SO}_{2}$ is assumed to have been obtained

$$
\begin{aligned}
& A(\lambda) \\
& \quad G_{0}+\left(\Delta \varepsilon_{1} \mathrm{SO}_{2}+\Delta \varepsilon_{2} \mathrm{SCO}+\varepsilon_{\mathrm{Hb}}(\lambda)\right) d_{0} T+G_{1} \lambda \\
& \quad+\lambda \exp \left(-\left(\Delta \varepsilon_{1} \mathrm{SO}_{2}+\Delta \varepsilon_{2} \mathrm{SCO}+\varepsilon_{\mathrm{Hb}}(\lambda)\right) d_{1} T\right) .
\end{aligned}
$$

Table 1. Different combinations of percent blood $\mathrm{OxyHb}\left(\mathrm{SO}_{2}\right)$ and $\mathrm{COHb}$ saturation $(\mathrm{SCO}), \mathrm{c}_{x}$, to give dermis absorption coefficients used in post Monte Carlo stage.

\begin{tabular}{lcccccccccccccccc}
\hline \multicolumn{1}{c}{$\mathrm{c}_{x}$} & $\mathrm{c}_{1}$ & $\mathrm{c}_{2}$ & $\mathrm{c}_{3}$ & $\mathrm{c}_{4}$ & $\mathrm{c}_{5}$ & $\mathrm{c}_{6}$ & $\mathrm{c}_{7}$ & $\mathrm{c}_{8}$ & $\mathrm{c}_{9}$ & $\mathrm{c}_{10}$ & $\mathrm{c}_{11}$ & $\mathrm{c}_{12}$ & $\mathrm{c}_{13}$ & $\mathrm{c}_{14}$ & $\mathrm{c}_{15}$ & $\mathrm{c}_{16}$ \\
\hline $\mathrm{SO}_{2}(\%)$ & 100 & 95 & 95 & 90 & 90 & 85 & 85 & 80 & 80 & 75 & 70 & 65 & 60 & 55 & 50 & 45 \\
$\mathrm{SCO}(\%)$ & 0 & 3 & 5 & 5 & 10 & 10 & 15 & 10 & 20 & 25 & 30 & 35 & 40 & 45 & 50 & 55 \\
\hline
\end{tabular}




\section{Results}

The Monte Carlo simulated reflectance data for a two-layered medium with optical properties described in Sec. 2.1 are used here to compare the accuracy of percent $\mathrm{OxyHb}$ and $\mathrm{COHb}$ saturation given by MLB and extended MLB. The estimation of $\mathrm{SO}_{2}$ and SCO value using MLB is presented here first. The estimation begins by substituting $\mu_{\mathrm{a}_{\mathrm{b}}}$ in Eq. (4) into $\mu_{\mathrm{a}}$ in Eq. (5) to give an expression of attenuation as a linear function of $\mathrm{SO}_{2}$ and $\mathrm{SCO}$. The fitting procedure described in Sec. 2.2 is then used to search for the best estimates of these parameters using the MLB. The difference between the actual and estimated percent $\mathrm{SO}_{2}$ and $\mathrm{SCO}$ value represented by $\Delta \mathrm{SO}_{2}$ and $\Delta \mathrm{SCO}$, respectively, is defined as:

$$
\begin{aligned}
\Delta \mathrm{SO}_{2} & =\mathrm{SO}_{2_{\mathrm{EST}}}-\mathrm{SO}_{2_{\mathrm{ACT}},} \\
\Delta \mathrm{SCO} & =\mathrm{SCO}_{\mathrm{EST}}-\mathrm{SCO}_{\mathrm{ACT}},
\end{aligned}
$$

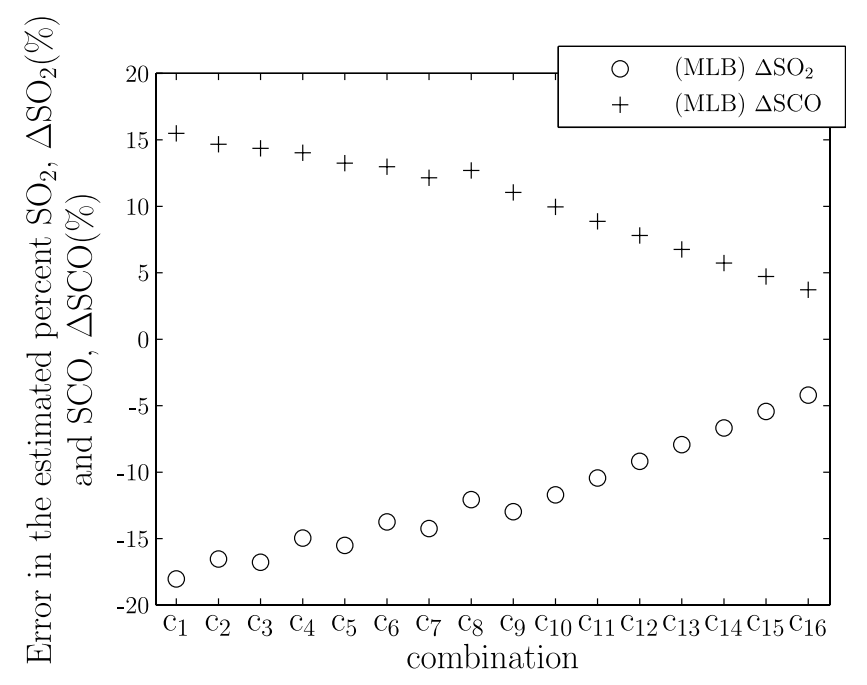

(a) $f_{\mathrm{v} \cdot \mathrm{mel}}=2 \%$

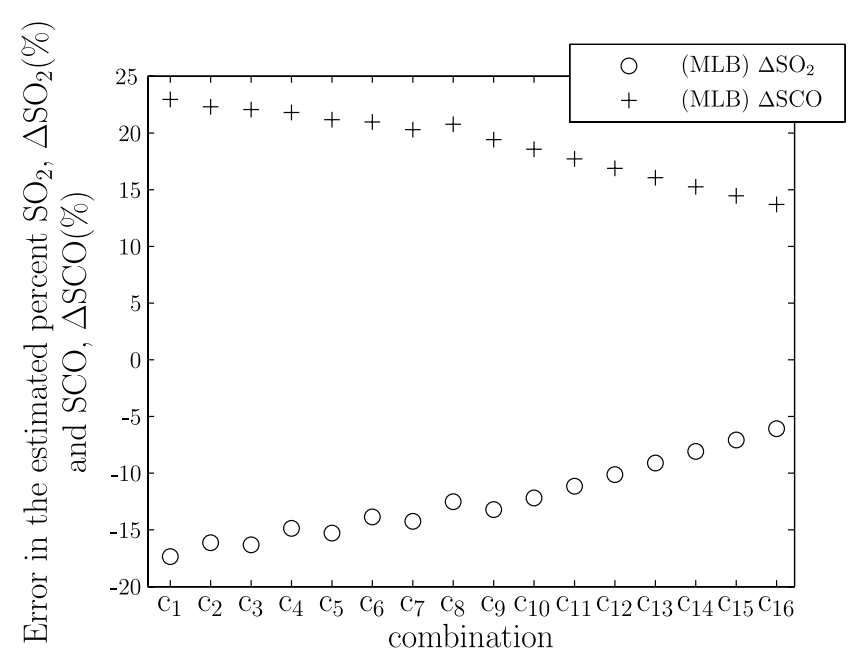

(b) $f_{\mathrm{v} \cdot \mathrm{mel}}=40 \%$

Fig. 2. Error in blood $\mathrm{OxyHb}$ saturation $\left(\Delta \mathrm{SO}_{2}\right)$ and blood $\mathrm{COHb}$ saturation $(\Delta \mathrm{SCO})$ given by MLB for different combinations of $\mathrm{SO}_{2}$ and SCO in Table 1 and when volume fraction of melanosomes $\left(f_{\mathrm{v} \cdot \mathrm{mel}}\right)$ is (a) $2 \%$ and (b) $40 \%$.

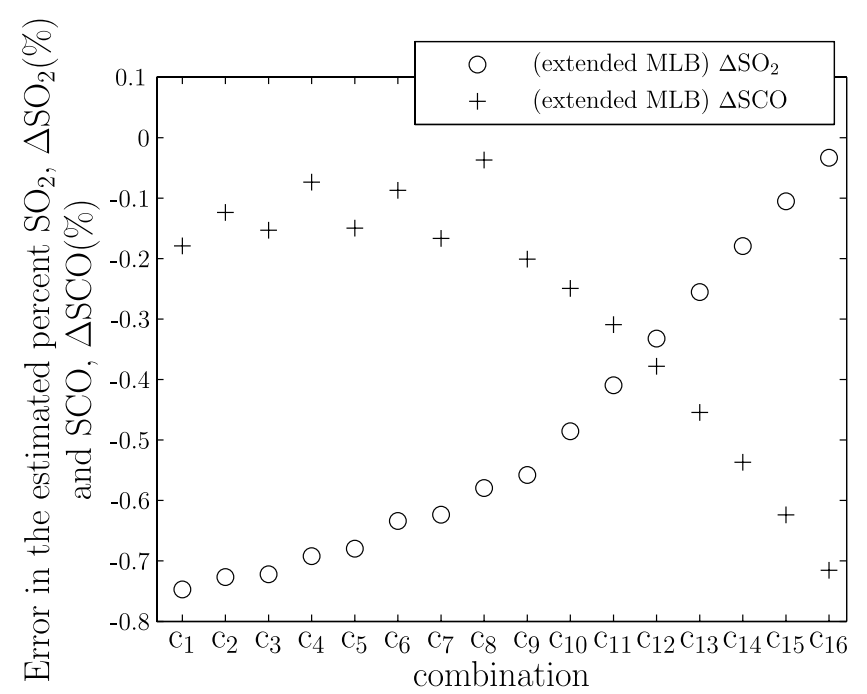

(a) $f_{\mathrm{v} \cdot \mathrm{mel}}=2 \%$

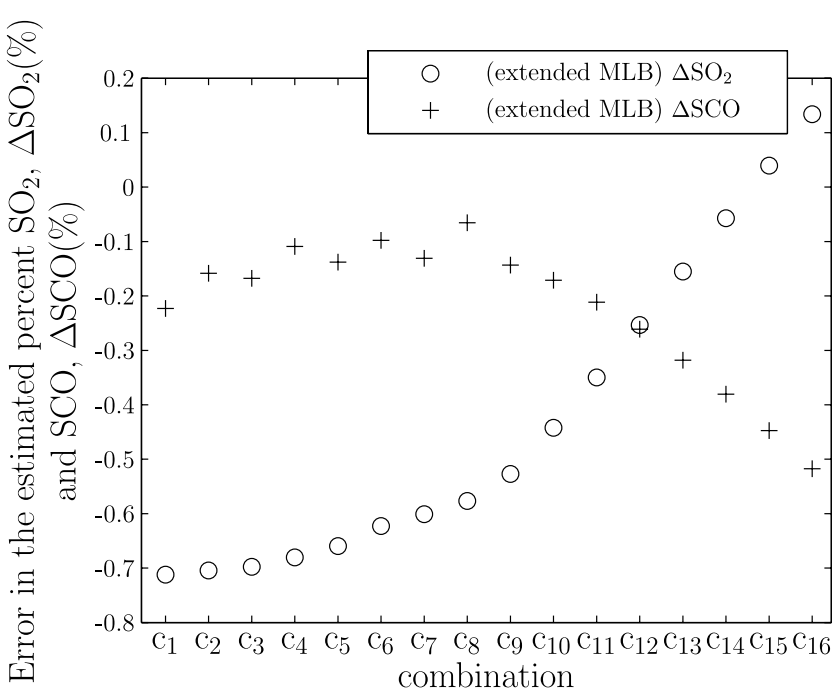

(b) $f_{\mathrm{v} \cdot \mathrm{mel}}=40 \%$

Fig. 3. Error in blood OxyHb saturation $\Delta \mathrm{SO}_{2}$ ("o") and blood COHb saturation $\Delta \mathrm{SCO}$ ("+") estimated by extended MLB when volume fraction of melanosomes ( $\left.f_{\mathrm{v} \cdot \mathrm{mel}}\right)$ is (a) $2 \%$ and (b) $40 \%$. The actual $\mathrm{SO}_{2}$ and SCO used in Monte Carlo simulations are listed in Table 1. 
where $\mathrm{SO}_{2_{\mathrm{EST}}}$ and $\mathrm{SCO}_{\mathrm{EST}}$ indicate that of the value estimated by the employed model while the actual percent $\mathrm{SO}_{2}$ and $\mathrm{SCO}$ value listed in Table 1 are symbolized by $\mathrm{SO}_{2_{\mathrm{ACT}}}$ and $\mathrm{SCO}_{\mathrm{ACT}}$. The $\Delta \mathrm{SO}_{2}$ and $\triangle \mathrm{SCO}$ given from the MLB against different combinations of $\mathrm{SO}_{2_{\mathrm{ACT}}}$ and $\mathrm{SCO}_{\mathrm{ACT}}$ in Table 1 and at $f_{\mathrm{v} \cdot \mathrm{mel}}$ of $2 \%$ and $40 \%$ are represented by symbol "o" and "+", respectively, and plotted in Figs. 2(a) and $2(\mathrm{~b})$.

An investigation of the accuracy of the estimates using extended MLB as compared to that of MLB is by using the same reflectance data and fitting method that are used to test the performance of the MLB in Fig 2. The best guess of percent $\mathrm{SO}_{2}$ and SCO at different combinations in Table 1 given from extended MLB in Eq. (7) is then used to calculate $\Delta \mathrm{SO}_{2}$ and $\Delta \mathrm{SCO}$ from Eqs. (8) and (9), and are plotted in Fig. 3.

\section{Discussion}

The comparison between the accuracy of values estimated by MLB and extended MLB in Figs. 2 and 3 shows a higher error in the values given by the MLB. The calculated mean absolute error is approximately $10 \%$ as compared to $0.4 \%$ that is produced by the extended MLB. This is likely due to the linear and single-valued light attenuation and blood absorption relationship that is assumed by the MLB. Furthermore, light absorption by melanin has not been considered in this model.

The error in the $\mathrm{SCO}$ and $\mathrm{SO}_{2}$ value determined by MLB in Fig. 2 is, in general, decreased with the combination index shown in Table 1 . Besides the result also showed the consistent overestimation of $\mathrm{SCO}$ and underestimation of $\mathrm{SO}_{2}$ value at different $f_{\mathrm{v} \cdot \mathrm{mel}}$ and combination index. An investigation of the attenuation spectrum of these $\mathrm{SO}_{2}$ and $\mathrm{SCO}$ combinations reveals that two hemoglobin peaks in the considered wavelength range of $520-600 \mathrm{~nm}$, which is attributed to the unique absorption features of OxyHb in Fig. 1, are apparent when $\mathrm{SO}_{2}=100 \%$ and $\mathrm{SCO}=0 \%$. These peaks gradually diminished with a decrease in $\mathrm{SO}_{2}$ (or an increase in $\mathrm{SCO}$ ). A possible explanation of the observed error pattern is that the MLB fails to sufficiently describe light attenuation contributed by underlying absorption (by different absorbers) and scattering processes, producing local minimum of the function that is not globally optimal during the fitting process when the hemoglobin peaks are at their highest. Another interesting fact that can be observed in Fig. 2 is the inconsistent magnitude of $\Delta \mathrm{SCO}$ given by the MLB as the $f_{\mathrm{v} \cdot \mathrm{mel}}$ varied. This could be due to the reason that an increase in epidermal light absorption reduces the hemoglobin peaks, which has the similar effect to that when the percent blood $\mathrm{COHb}$ saturation increases, leading to an inconsistent error in SCO given by the MLB for measurement on different pigmented skin.

The considerably consistent error in the $\mathrm{SO}_{2}$ and SCO value estimated by extended MLB across different volume fraction of melanosomes in Fig. 3 with the mean of their differences in absolute $\Delta \mathrm{SO}_{2}$ and $\Delta$ SCO given by $0.03 \%$ and $0.056 \%$, respectively, implies that the effects of epidermal melanin absorption on the measured attenuation are reasonably accurately represented by the wavelength dependent linear term in the developed model in Eq. (7). Even though the exponential term in this model is to account for inseparable effects of light scattering and absorption in the dermis on the measured signals, there is an overall slight underestimate of $\mathrm{SO}_{2}$ and $\mathrm{SCO}$ values in Figs. 3(a) and 3 (b) independent of $f_{\text {v.mel }}$ value. This could be resulted from the insufficiency of this model at describing light propagation behavior in the dermis layer of the skin model. These errors are, however, considerably small $(< \pm 1 \%)$, suggesting the ability of this model to resolve the light attenuation contributed by the different absorption of the present absorbers. Since this study worked with signals in the wavelength range of $520-600 \mathrm{~nm}$, wherein the variation in absorption characteristics of dermal absorbers in Fig. 1 is most distinctive, the low errors shown in Fig. 3 confirm the suitability of the corresponding wavelengths for quantification works using the developed extended MLB. It should be noted that the use of a narrower wavelength range such as that of $520-580 \mathrm{~nm}$ which contains the corresponding absorption spectra variation will produce the same result.

The viability of using extended MLB and the described strategy to determine blood $\mathrm{SO}_{2}$ and SCO with reasonable accuracy indicates that this can be a suitable means for fast and continuous monitoring of one's percentage $\mathrm{OxyHb}$ and $\mathrm{COHb}$, and in vivo experiment works are currently underway to confirm this. The technique described here does not depend on the calibrated data as is required in pulse oximetry and would be insensitive to noise on signals in the measurement of $\mathrm{SO}_{2}$ and 
SCO. Furthermore, the effects of light absorption by melanin has been accounted for in this developed model, hence accuracy of the measurements would not be compromised by differences in skin color. This renders the discussed fitting technique and model potentially to be used particularly for $\mathrm{CO}$ poisoning diagnosis and treatment, or as a cost effective health care tool used on power plant workers and firefighters who are at risk of flammable and toxic gas exposure.

\section{Conclusion}

This study demonstrates the use of extended MLB law model for non-invasive and continuous monitoring of percentage saturation of oxygen and $\mathrm{CO}$ in blood. This work concludes that accurate measurement of these values can be obtained if the developed fitting model has sufficiently described the true underlying light absorption and scattering processes. In addition, wavelengths in the range of 520$600 \mathrm{~nm}$ is shown to be suitable as working wavelengths for the estimation of blood $\mathrm{SO}_{2}$ and $\mathrm{SCO}$ using $\mathrm{CW}$ spectroscopic measurement data.

\section{References}

1. A. L. Ilano, T. A. RaffinKienle, "Management of carbon monoxide poisoning," Chest 97, 165-169 (1990).

2. M. Touger, A. Birnbaum, J. Wang, K. Chou, D. Pearson, P. Bijur, "Performance of the RAD-57 Pulse Co-oximeter compared with standard laboratory carboxyhemoglobin measurement," $\mathrm{Am}$. College of Emergency Phys. 56(4), 382-388 (2010).

3. G. Zhang, A. Katz, R. R. Alfano, A. D. Kofinas, D. A. Kofinas, P. G. Stubblefield, W. Rosenfeld, D. Beyer, D. Maulik, M. R. Stankovic, "Brain perfusion monitoring with frequency-domain and continuous-wave near-infrared spectroscopy: A cross-correlation study in newborn piglets," Phys. Med. Biol. 45, 3143-3158 (2000).

4. A. Vogel, V. V. Chernomordik, J. D. Riley, M. Hassan, F. Amyot, B. Dasgeb, S. G. Demos, R. Pursley, R. F. Little, R. Yarchoan, Y. Tao, A. H. Gandjbakhche, "Using noninvasive multispectral imaging to quantitatively assess tissue vasculature," J. Biomed. Opt. 12(5), 051604 (2007).
5. A. Pifferi, P. Taroni, G. Valentini, S. AnderssonEngels, "Real-time method for fitting time-resolved reflectance and transmittance measurements with a Monte Carlo Model," Appl. Opt. 37(13), 2774-2780 (1998).

6. M. Kobayashi, Y. Ito, N. Sakauchi, I. Oda, I. Konishi, Y. Tsunazawa, "Analysis of nonlinear relation for skin hemoglobin imaging," Opt. Exp. 9(13), 802-812, (2001).

7. D. Yudovsky, L. Pilon, "Rapid and accurate estimation of blood saturation, melanin content, and epidermis thickness from spectral diffuse reflectance," Appl. Opt. 49(10), 1707-1719 (2010).

8. I. V. Meglinski, S. J. Matcher, "Quantitative assessment of skin layers absorption and skin reflectance spectra simulation in the visible and near-infrared spectral regions," Physiol. Measurement 23(4), 741753 (2002).

9. T. Maeda, N. Arakawa, M. Takahashi, Y. Aizu, "Monte Carlo simulation of spectral reflectance using a multilayered skin tissue model," Opt. Rev. 17(3), 223-229 (2010).

10. A. Kienle, R. Hibst, "A new optimal wavelength for treatment of port wine stains?" Phys. Med. Biol. 40(10), 1559-1576 (1996).

11. H. J. Van Staveren, C. J. M. Moes, J. Van Marle, M. J. C. Van Gemert, S. A. Prahl, "Light scattering in Intralipid-10 in the wavelength range of 4001100 nm," Appl. Opt. 30, 4507-4514 (1991).

12. A. N. Bashkatov, E. A. Genina, V. I. Kochubey, V. V. Tuchin, "Optical properties of human skin, subcutaneous and mucous tissues in the wavelength range from 400 to $2000 \mathrm{~nm}, "$ J. Phys. Appl. Phys. 38, 2543-2555 (2005).

13. S. L. Jacques, D. J. McAuliffe, "The melasome: Threshold temperature for explosive vaporization and internal absorption coefficient during pulsed laser irradiation," Photochem. Photobiol. 53, 769-775 (1991).

14. W. G. Zijlistra, A. Buursma, O. W. Van Assendelft, Visible and Near Infrared Absorption Spectra of Human and Animal Haemoglobin: Determination and Application, VSP International Science Publisher, Netherlands (2000), pp. 180-194.

15. P. C. Y. Chang, J. G. Walker, K. I. Hopcraft, B. Ablitt, E. Jakeman, "Polarization discrimination for active imaging in scattering media," Opt. Commun. 159, 1-6 (1999).

16. R. N. Pittman, B. R. Duling, "A new method for the measurement of percent oxyhemoglobin," J. Appl. Physiol. 38(2), 315-320 (1975). 